\title{
Maximum Value Derivation Technique
}

National Cancer Institute

\section{Source}

National Cancer Institute. Maximum Value Derivation Technique. NCI Thesaurus. Code C82868.

A data derivation technique used to calculate a subject's maximum value over a defined set of records. 